\title{
A randomized controlled trial comparing early versus late oral feeding after cesarean section under regional anesthesia
}

This article was published in the following Dove Press journal: International Journal of Women's Health

\section{Apinun Luksanachinda Mawson (iD \\ Sommart Bumrungphuet (iD Jittima Manonai $(\mathbb{D}$}

Department of Obstetrics and Gynecology, Faculty of Medicine, Ramathibodi Hospital, Mahidol University, Bangkok, Thailand
Correspondence: Sommart Bumrungphuet

Department of Obstetrics and Gynecology, Faculty of Medicine, Ramathibodi Hospital, Mahidol University, 270 Rama VI Road, Ratchathewi, Bangkok 10400, Thailand

Tel $+662201 \quad 1412$

Email sommart.bum@mahidol.ac.th
Objective: To compare the return of bowel movements in regionally anesthetized women undergoing cesarean section (C-section) given Early Oral Feeding (EOF) to that of women given Late Oral Feeding (LOF). Secondary outcomes of maternal satisfaction and gastrointestinal complications were also examined.

Methods: In a single-blinded randomized controlled trial (TCTR20181202001), 148 singleton pregnant women undergoing elective $\mathrm{C}$-sections with regional anesthesia were assigned to receive either EOF or LOF. Participants began to sip water at 6-8 hrs or more than $12 \mathrm{hrs}$ post-operation, for EOF or LOF respectively. Participants were then placed onto a stepping diet as tolerated. Participants failing to tolerate the stepping diet or those having surgical complications were excluded from the study.

Results: After exclusion, 69 women remained in the EOF group and 67 in the LOF group. The ages of participants ranged from 19 to 42, with a mean of 32.07. There was no-loss follow up and no significant difference in patient characteristics, except the site of the surgical incision. Participants given EOF were more likely to experience bowel sound the next morning than patients given LOF (EOF $87.0 \%$, LOF $44.8 \%, P$-value $<0.001$ ). However, there was no difference in time to passing flatus and time to passing stool. Maternal satisfaction regarding hunger (EOF 3.78 \pm 0.91 , LOF $3.24 \pm 1.01, P$-value 0.002 ) and maternal satisfaction with postoperative consumption (EOF $4.38 \pm 0.64$, LOF $4.13 \pm 0.48, P$-value $0.049)$ were significantly higher in the EOF group. There was no difference in gastrointestinal complications between the groups ( $P$-value 0.978).

Conclusion: The EOF group experienced an earlier return of bowel movement and greater maternal satisfaction than the LOF group, with no difference in gastrointestinal complications. These findings support the recommendation of EOF for women who undergo uncomplicated C-sections under regional anesthesia.

Keywords: early oral feeding, cesarean section, maternal satisfaction, bowel function, gastrointestinal complication, randomized controlled trial

\section{Introduction}

Cesarean section (C-section) is the most common surgery in the world ${ }^{1}$ and is defined as a laparotomy followed by a hysterotomy and fetal delivery. The current global standard is to use regional anesthesia which allows patients to remain awake which minimizes drug transfer to the fetus. In addition, this procedure allows practitioners to deliver opioid through an intrathecal route, thereby decreasing the risk of choking and difficult intubation. ${ }^{2}$ Intrathecal morphine (duration of action 
$24 \mathrm{hr}$.) is the most common analgesic used in elective C-sections; however, side effects can include nausea, vomiting, skin irritation, ${ }^{3,4}$ and increased lag time of gastric emptying. ${ }^{5}$ Even though current $\mathrm{C}$-section procedures are less complicated and involve shorter hospital stays than in the past, ${ }^{2}$ several postoperative complications, such as ileus $9.3 \%$, nausea $4.6 \%$ and vomiting $2.4 \%$, commonly occur. ${ }^{6,7}$

Following abdominal surgery, activity in small intestines starts within 2-3 hrs and function is completely recovered within 6-12 hrs. Stomach function returns 12$24 \mathrm{hrs}$ after surgery with the large intestines recovering fully between 48-72 hrs. ${ }^{8}$ Bowel sound (examined by a physician), passing flatus, and bowel movement, ${ }^{9}$ provide clinicians with key indicators to gauge the return of bowel function. Factors influencing these metrics include the size of the incision, the surgical site, operative time, blood loss, type of anesthesia, opioids, general health of the patient, nutrition, and psychiatric condition. ${ }^{8}$

A typical C-section patient at Ramathibodi Hospital, Bangkok, lies down flat following the procedure for at least $6 \mathrm{hrs}$ or until digestive function has recovered (usually 12-24 hrs after surgery). Patients will begin sipping water the morning after surgery and progresses to a stepping diet consisting of a liquid diet for the first meal, a soft diet for the next, then a regular diet at each following meal. IV fluid is discontinued as soon as the patient resumes eating. At this time the urine catheter can be removed and the patient is advised to ambulate. The patient is typically discharged $72 \mathrm{hrs}$ after surgery.

A review of the literature reveals that early oral feeding can minimize protein depletion or destruction in the body, aid healing of the surgical wound, improved mental-state, ${ }^{10}$ reduced sensation of thirst and hunger, ${ }^{11,12}$ and reduced post-operative pain. $^{13}$ Other benefits of early feeding include improved recovery of bowel function, decreased time to lactation, ${ }^{14}$ decreased abdominal bloating, ${ }^{10,15}$ decreased time to pass flatus ${ }^{6,13,14,16-18}$ or stool, ${ }^{11,14}$ reduced number of IV bags used, ${ }^{11,12,14-16}$ reduced time for removal of urine catheter, ${ }^{12}$ shortened time to ambulation $^{12,14,18}$ and discharge, ${ }^{6,11,14}$ and improved overall satisfaction with the surgery. ${ }^{6,7,10-18}$ These benefits are realized without gastrointestinal complications. ${ }^{7,10-13,15-19}$

While support for advising EOF can be found in the literature (especially meta-analysis study by Guo et al and RCT study by Saad et. al), specific recommendations regarding the ideal time to begin feeding have not been clearly established. The primary outcome investigated in this study was the impact of EOF on the return of bowel function. Secondary outcomes evaluated were time to passing stool and flatus, maternal satisfaction, and post-operative consumption. Although it is beyond the scope of this particular study, developing a "best-practices" set of timing recommendations for the post-C-section stepping diet is a logical extension to the current investigation and one which could represent a valuable contribution to the knowledge-base.

\section{Materials and methods Study type, setting, and duration}

A single-blinded randomized controlled study was conducted under the intention-to-treat protocol at the Department of Obstetrics \& Gynecology, Faculty of Medicine, Ramathibodi Hospital, Mahidol University. Between April 2018 and December 2018 one hundred and forty-eight pregnant women undergoing uncomplicated elective $\mathrm{C}$-section in the obstetrics ward were enrolled in the study. The study protocol was approved by the Committee on Human Rights Related to Research Involving Human Subjects, Ramathibodi Hospital on 14 March 2018. The study was approved by the Thai Clinical Trials Registry, RCT number “TCTR20181202001". Written informed consent was given by all participants before the study enrollment. This trial was conducted in accordance with the Declaration of Helsinki.

\section{Study participants}

The inclusion criteria defined eligible participants as singleton pregnant women who underwent elective $\mathrm{C}$-section under regional anesthesia, desire to join the study after being giving research information, did not have another surgery except tubal ligation and denied a history of gastrointestinal surgery or underlying diseases that affect digestion. Women that needed to delay postoperative oral feeding more than 6-12 hrs (due to severe preeclampsia or late postpartum hemorrhage) were excluded. Women who had intraoperative and postoperative complications such as gastrointestinal injury, genitourinary tract injury, and postpartum hemorrhage $(\mathrm{PPH})$ were also excluded.

Sample size calculation

The statistical formula used to calculate minimal sample size is shown below.

$$
n_{t r t}=\frac{\left(z_{1-\frac{\alpha}{2}}+z_{1-\beta}\right)^{2}\left[\sigma_{t r t}^{2}+\frac{\sigma_{c o n}^{2}}{r}\right]}{\Delta^{2}}
$$




$$
r=\frac{n_{c o n}}{n_{t r t}}, \Delta=\mu_{t r t}-\mu_{c o n}
$$

Where, $\mathrm{n}_{\text {trt }}=$ minimum sample size, ratio $(\mathrm{r})=1, \alpha$ determined Type I (alpha) error $=0.05, \beta$ determined Type II (beta) error or power $=0.2(80 \%)$. The meta-analysis study by Guo et $\mathrm{al}^{22}$ provides a mean time to passing flatus of $20.18 \mathrm{hrs}$ for EOF group, represented as $\mu_{\text {trt }}$, and $24.12 \mathrm{hrs}$ in LOF group, represented as $\mu_{\text {con }}$. Based on the above parameters, we determined a minimum sample size of 148 participants to achieve a statistically significant result. Therefore, the sample size for this study is 148 divided into 2 groups of 74 .

\section{Randomization}

The randomized allocation was performed using a computerized random number generator with a block of four restriction. The resulting identifiers were placed in order in sealed envelopes by the clinical statistician. Envelopes were attached to doctor's order sheets and were opened following surgery and the information was personally copied onto their clinicians' order sheets. Oral feedings were given to the participants by nurses. Single blinding of the analyzer was performed; however, participants and nurses were not blinded. The blinded assessor was the same individual as the investigator who distributed the informed consent and the analyzer.

\section{Intervention}

The participants were randomly allocated to receive either EOF or LOF. The EOF group was assigned to start sipping water at 6-8 hrs after surgery then progress to a soft diet and regular diet in the next meals, as tolerated. The LOF group followed the same pattern but began sipping water at more than $12 \mathrm{hrs}$ post-operation. All participants were advised and signed informed consent forms. C-sections were performed by residents or instructors and the regional anesthesia, with or without intrathecal morphine, was administered by anesthesiologists using the standard protocol.

\section{Study outcomes}

The primary outcome of this study is the assessment of the return of bowel function after $\mathrm{C}$-section, as gauged by time to pass flatus, time to pass stool, and the presence of bowel sounds the morning after surgery. Secondary outcomes are the evaluation of maternal satisfaction and GI complications, defined as mild ileus symptoms and severe ileus symptoms. The presence of anorexia, abdominal cramping, or mild distension on physical examination is defined as mild ileus symptoms. Marked abdominal distension with more than 3 episodes of vomiting in $24 \mathrm{hrs}$ after surgery, or the inability to tolerate a liquid diet with delayed step diet is defined as severe ileus symptoms.

In the post-partum ward following surgery, assessment forms were given to the participants who recorded gastrointestinal complications (these included nausea, vomiting, and abdominal bloating), time to passing flatus, time to passing stool, time to lactation, time to removing IV fluids, time to removing urine catheter, time to ambulation, and length of hospital stay. Oral feeding was administered according to doctor orders by nurses in the post-partum ward. Bowel sound was examined the morning after surgery. ${ }^{20}$ On the day of discharge maternal satisfaction with thirst, hunger, postoperative consumption, and postoperative care were assessed using a 5-point Likert scale. ${ }^{21}$ The study results were collected by the blinded assessor. This same individual also handed out the forms and examined bowel sound.

\section{Statistical analysis}

Data were analyzed by IBM SPSS statistics 17.0 and all outcomes were tested for normality. For normally distributed data, two independent engines performed Student's $t$-tests on the continuous data and generated the mean and standard deviation (SD). The category data was analyzed using a Chi-square test. Results not following a normal distribution were analyzed using a Mann-Whitney $U$ test. $P$-values below 0.05 were defined as statistically significant.

\section{Results}

A total of 148 participants were enrolled in this study according to the inclusion criteria. Seventy-four women were randomly assigned to either the EOF or the LOF group. Twelve people were excluded due to PPH (10 cases) and genitourinary injury (2 cases) after C-section. Results were determined based on data from the 69 participants remaining in the EOF group and the 67 remaining in the LOF group. A diagram of the research design is presented in Figure 1, showing the randomization design for selection and allocation, and the criteria evaluated. The ages of participants ranged from 19-42 years, with a mean of 32.07. Participants were observed from initial recruitment until discharge. 


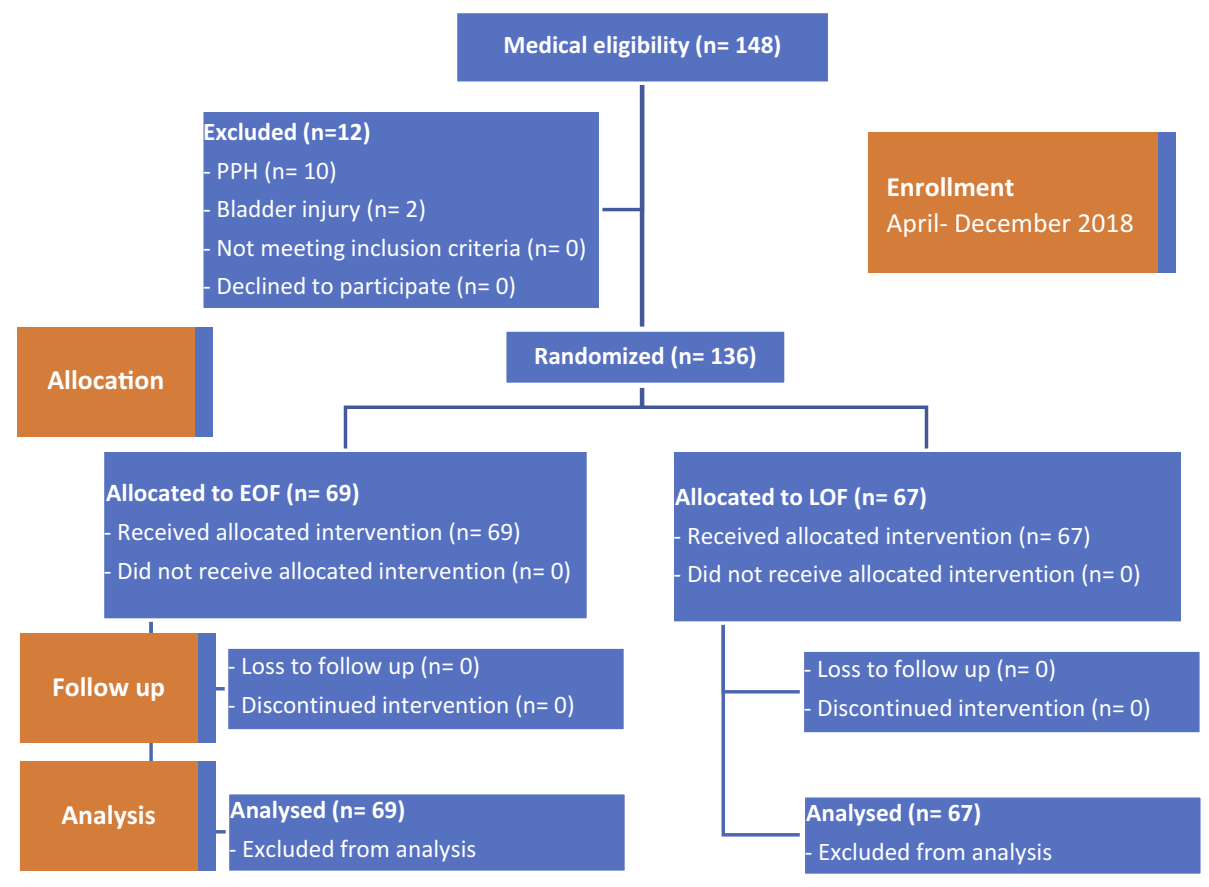

Figure I Randomization diagram.

The main significant difference in the characteristics of participants was the surgical incision (Pfannenstiel incision in EOF group $91.2 \%$ and LOF group $97.1 \%, P$-value 0.016). Factors such as maternal age, gestational age, parity, pre-pregnancy BMI, surgeons, history of C-section, intrathecal morphine, operative time, and estimated blood loss were not statistically different between the EOF and LOF groups, as seen in Table 1.

Outcomes are shown in Table 2. EOF resulted in increased bowel sound the morning after surgery, EOF $87.0 \%$ and LOF $44.8 \%$ ( $P$-value $<0.001)$. However, no difference in time to passing flatus (EOF 1,589.4 $\pm 802.8 \mathrm{~min}, \mathrm{LOF} 1,621.8 \pm 756.6, P$-value 0.809$)$ or time to passing stool after surgery (EOF 3,213 $\pm 868.8 \mathrm{~min}$, LOF $3,084 \pm 660, P$-value 0.504$)$ were observed. Maternal satisfaction regarding hunger (EOF 3.78 \pm 0.91 , LOF $3.24 \pm 1.01$, $P$-value 0.002$)$ and maternal satisfaction with postoperative consumption (EOF 4.38 \pm 0.64 , LOF $4.13 \pm 0.48$, $P$-value 0.049$)$ were significantly greater in the EOF group. There was no difference in gastrointestinal complications (EOF $42.03 \%$, LOF $41.79 \%, P$-value 0.978 ). Other complications monitored, nausea, vomiting and bloating at 6-8 hrs after surgery, $24 \mathrm{hrs}$ after surgery and at discharge were also not significantly different ( $P$-value $\geq 0.05$ ).

In summary, our results indicate that EOF improves bowel function and maternal satisfaction after C-section without increasing post-operative complications.

\section{Discussion}

In this study, data for the return of bowel function following uncomplicated $\mathrm{C}$-section under regional anesthesia was evaluated in the EOF and LOF groups. This comparative evaluation revealed that EOF is superior to LOF. Firstly, EOF increased bowel sound (a key indicator of the return of bowel function) the morning after surgery. Secondly, there was no difference in GI complications between the EOF and LOF groups. Finally, EOF increased maternal satisfaction.

Other studies also support the advisability of EOF, with a variety of benefits identified including the time to passing flatus, ${ }^{6,13,14,16-18}$ time to return of bowel sound, time to passing stool,,$^{71-18}$ and time to bowel movement. Our findings confirm that EOF increases bowel sound the morning after surgery; however, our results did not demonstrate significant differences between EOF and LOF groups in time to passing flatus and time to passing stool. A lack of statistically significant variation in these factors may be due to the homogeneity in the characteristics of our population. Our sample consisted of low-risk, elective C-section recipients with similar operative times, lacks variation in terms of the quantity and severity of significant factors contributing to ileus. With factors contributing to ileus similar and few, and the likelihood of complications low, 
Table I Characteristics of participants in EOF and LOF groups after C-section under regional anesthesia

\begin{tabular}{|c|c|c|c|c|}
\hline Characteristics & Total $(n=136)$ & Early Feeding $(n=69)$ & $\begin{array}{l}\text { Late Feeding } \\
(n=67)\end{array}$ & P-Value \\
\hline Maternal Age (year) ${ }^{\mathrm{a}}$ & $32.1 \pm 11.3$ & $32.3 \pm 5.0$ & $31.8 \pm 5.6$ & 0.544 \\
\hline Gestational Age (weeks) ${ }^{\mathrm{a}}$ & $38.4 \pm 0.6$ & $38.4 \pm 0.6$ & $38.3 \pm 0.6$ & 0.553 \\
\hline $\begin{array}{l}\text { Parity }(\%)^{b} \\
\cdot 0 \\
\cdot 1 \\
\cdot \geq 2\end{array}$ & $\begin{array}{l}22.1 \\
62.5 \\
15.4\end{array}$ & $\begin{array}{l}21.7 \\
60.9 \\
17.3\end{array}$ & $\begin{array}{l}22.4 \\
64.2 \\
13.5\end{array}$ & 0.633 \\
\hline $\begin{array}{l}\text { Prepregnant BMI }\left(\mathrm{kg} / \mathrm{m}^{2}\right)^{\mathrm{a}} \\
\cdot<18.5(\%) \\
\cdot 18.5-24.9(\%) \\
\cdot 25-29.9(\%) \\
\cdot \geq 30(\%)\end{array}$ & $\begin{array}{l}23.6 \pm 4.6 \\
10.3 \\
61 \\
16.9 \\
11.8\end{array}$ & $\begin{array}{l}23.8 \pm 4.3 \\
5.8 \\
66.7 \\
17.4 \\
10.1\end{array}$ & $\begin{array}{l}23.4 \pm 5.0 \\
14.9 \\
55.2 \\
16.4 \\
13.4\end{array}$ & 0.641 \\
\hline $\begin{array}{l}\text { Indication for C-section (\%) } \\
\text { - Previous C-section } \\
\text { - Malpresentation } \\
\text { - CPD } \\
\text { - Other }\end{array}$ & $\begin{array}{l}77.9 \\
12.5 \\
2.9 \\
6.7\end{array}$ & $\begin{array}{l}76.8 \\
13.0 \\
2.9 \\
7.3\end{array}$ & $\begin{array}{l}79.1 \\
11.9 \\
3 \\
6\end{array}$ & 0.965 \\
\hline 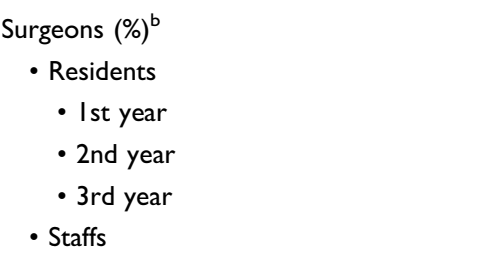 & $\begin{array}{l}14 \\
55.9 \\
27.9 \\
2.2\end{array}$ & $\begin{array}{l}11.6 \\
55.2 \\
10.4 \\
2.9\end{array}$ & $\begin{array}{l}16.4 \\
56.7 \\
25.4 \\
1.5\end{array}$ & 0.751 \\
\hline $\begin{array}{l}\text { Surgical site }(\%)^{\mathrm{b}} \\
\text { - Pfannenstiel } \\
\text { - Midline }\end{array}$ & $\begin{array}{l}91.2 \\
8.8\end{array}$ & $\begin{array}{l}97.1 \\
2.9\end{array}$ & $\begin{array}{l}85.1 \\
14.9\end{array}$ & 0.016 \\
\hline $\begin{array}{l}\text { History of C-section }(\%)^{\mathrm{b}} \\
\cdot \text { no } \\
\text { - Previous I time } \\
\text { - Previous } \geq 2 \text { times }\end{array}$ & $\begin{array}{l}22.1 \\
68.4 \\
9.5\end{array}$ & $\begin{array}{l}24.6 \\
63.8 \\
11.6\end{array}$ & $\begin{array}{l}19.4 \\
73.1 \\
7.5\end{array}$ & 0.218 \\
\hline $\begin{array}{l}\text { Intrathecal morphine }(\%)^{\mathrm{b}} \\
\text { - With } \\
\text { - Without }\end{array}$ & $\begin{array}{l}77.2 \\
22.8\end{array}$ & $\begin{array}{l}76.8 \\
23.2\end{array}$ & $\begin{array}{l}77.6 \\
22.4\end{array}$ & 1.000 \\
\hline Operative Time $(\mathrm{min})^{\mathrm{a}}$ & $59.1 \pm 14.0$ & $59.1 \pm 14.5$ & $59.0 \pm 13.4$ & 0.939 \\
\hline Estimated Blood Loss $(\mathrm{mL})^{\mathrm{a}}$ & $440.0 \pm 155.0$ & $578.7 \pm 163.8$ & $421.6 \pm 143.6$ & 0.164 \\
\hline Time to bowel sound examination $(\min )^{a}$ & $1,078.0 \pm 99.7$ & $1,073.5 \pm 96.9$ & $1,082.8 \pm 103.0$ & 0.589 \\
\hline
\end{tabular}

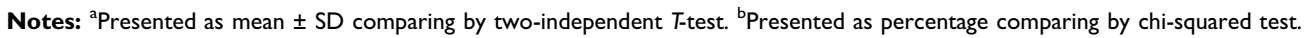

statistically significant variation is less likely to reveal itself, especially in studies with smaller sample sizes. However, our findings were generally consistent with those typically found in the literature.

Reports vary regarding the effects of EOF on maternal satisfaction. Guo et al's leading 2015 meta-analysis reported no link between EOF and general improvements in maternal satisfaction. ${ }^{19}$ In contrast, Teoh WHL et. al. in their 2007 study of Singaporean population, ${ }^{14}$ as well as this study, found that EOF participants enjoyed higher maternal satisfaction. Increased maternal satisfaction is an important outcome of care as it affects postpartum blues, decisions about future pregnancies, and doctor and hospital reputation. 
Table 2 Main outcomes in EOF and LOF groups after C-section under regional anesthesia

\begin{tabular}{|c|c|c|c|c|}
\hline Outcomes & Total $(n=136)$ & Early Feeding $(n=69)$ & Late Feeding $(n=67)$ & P-Value \\
\hline Time to Passing Flatus (min) & $1605.0 \pm 778.0$ & $1,589.4 \pm 802.8$ & $\mathrm{I}, 62 \mathrm{I} .8 \pm 756.6$ & 0.809 \\
\hline Present Bowel Sound at Next Morning (\%) & 66.2 & 87.0 & 44.8 & $<0.001$ \\
\hline Time to Passing Stool (min) ${ }^{\mathrm{a}}$ & $3031.0 \pm 976.0$ & $3,213.0 \pm 868.8$ & $3,084.0 \pm 660.0$ & 0.504 \\
\hline Mild ileus symptoms (\%) ${ }^{\mathrm{b}}$ & 41.9 & 42.0 & 41.8 & 0.978 \\
\hline - Nausea at 6-8 hr. & 4.4 & 5.8 & 3 & 0.681 \\
\hline - Nausea within $24 \mathrm{hr}$. & 7.3 & 7.2 & 7.5 & 0.870 \\
\hline - Nausea at discharge & 0.7 & 0 & 1.5 & 0.493 \\
\hline - Vomiting at 6-8 hr. & 3.6 & 5.7 & 1.5 & 0.245 \\
\hline - Vomiting within $24 \mathrm{hr}$. & 5.1 & 2.8 & 7.5 & 0.189 \\
\hline - Vomiting at discharge & 0 & 0 & 0 & - \\
\hline - Bloating at 6-8 hr. & 9.6 & 8.7 & 10.4 & 0.777 \\
\hline - Bloating within $24 \mathrm{hr}$. & 27.2 & 27.5 & 26.9 & 1.000 \\
\hline - Bloating at discharge & 2.5 & 1.4 & 4.5 & 0.362 \\
\hline Severe ileus symptoms (\%) & 0 & 0 & 0 & - \\
\hline \multicolumn{5}{|l|}{ Satisfaction Level $(I-5)^{\mathrm{a}}$} \\
\hline • Hunger & $3.5 \pm 1.0$ & $3.8 \pm 0.9$ & $3.2 \pm 1.0$ & 0.002 \\
\hline • Thirst & $3.5 \pm 1.1$ & $3.6 \pm 1.0$ & $3.4 \pm 1.2$ & 0.269 \\
\hline - Postoperative consuming & $4.3 \pm 0.7$ & $4.4 \pm 0.6$ & $4.1 \pm 0.8$ & 0.049 \\
\hline - Postoperative care & $4.7 \pm 0.5$ & $4.7 \pm 0.4$ & $4.7 \pm 0.5$ & 0.776 \\
\hline
\end{tabular}

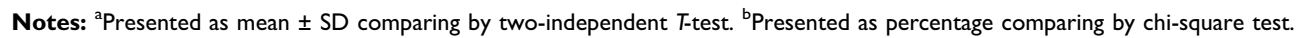

Previous studies are almost unanimously in confirming no difference in GI complications associated with EOF versus LOF, and this study is no exception. However, in a rare finding, the study by Teoh WHL et. al. 2007 reported increased nausea in their EOF participants, who began sipping orange-juice at only 30 mins post-operation. Despite the increased nausea $(10.2 \%$ vs $2 \%, P<0.05)$ experienced by EOF recipients, maternal satisfaction was clearly higher in the EOF group $(P<0.0001) .{ }^{14}$ It is possible that nausea experienced was easily treatable or too mild to detract from maternal satisfaction. Certainly, maternal satisfaction is affected by more severe nausea symptoms and GI complications which can cause postoperative pain, impede progress in the stepping diet, and increase the duration of hospital stay.

The strengths of our study are its research design. This investigation is a randomized controlled trial study with the assessor blinded (single blinding) and no-loss follow up. The participants filled-out their assessment surveys in real-time as they recovered in the post-partum ward, reducing the recall bias in participants.

However, this study has some limitations. The cases included in this investigation were elective and uncomplicated C-section. Thus, the results cannot be reliably applied to complicated or emergency C-section cases. Additionally, we could not blind the nurses and participants, only the assessor. Although this study was randomized into two groups, the surgical site (defined as Pfannenstiel and midline incision), remained significantly different with $97.1 \%$ of the EOF group and $85.1 \%$ of the LOF group receiving Pfannenstiel incision ( $P$-value $=0.016)$. There was no significant difference in postoperative pain and opioid consumption in women provided with either a midline or Pfannenstiel incision; ${ }^{23}$ however, the distance of the surgical site from abdomen does affect the duration of ileus and time to motility. ${ }^{8}$ It remains unclear whether receiving Pfannenstiel or midline incision produces predictable differences in outcome. Our experience and findings suggest that further studies should include a larger sample size, emergency cases, earlier oral feeding, and look more closely at the effects of the surgical site on key metrics.

\section{Conclusion}

Improvements in return of bowel function and maternal satisfaction, coupled with a lack of gastrointestinal complications, support the advisability of EOF over LOF. Looking forward, the extent to which the site of the surgical incision 
impacts key outcomes should be examined in more detail. Additionally, further research should consider beginning EOF earlier, should also include emergency cases, as well as other categories of $\mathrm{C}$-section patients where factors contributing to ileus are more severe and varied.

\section{Data sharing statement}

The datasets analyzed during the current study are available from the corresponding author on reasonable request.

\section{Acknowledgments}

The authors would like to thank the faculty, obstetrics residents and the nurses in Obstetrics and Post-partum ward at the Department of Obstetrics and Gynecology, Faculty of Medicine, Ramathibodi Hospital, Mahidol University, Bangkok. We also appreciate the statistical information provided by Umaporn Udomsubpayakul, Statistician, Section for Clinical Epidemiology and Biostatistics, Faculty of Medicine, Ramathibodi Hospital, Mahidol University, Bangkok and language correction provided by Matthew Brewster Mawson, owner of Master's English Laboratory, Bangkok and Marcia Brewster, Senior Consultant at Nautilus International Development Consulting, Inc. and former editor-in-chief, Natural Resources Forum.

\section{Disclosure}

The authors report no conflicts of interest in this work.

\section{References}

1. Betran AP, Merialdi M, Lauer JA, et al. Rates of cesarean section: analysis of global, regional and national estimates. Paediatr Perinat Epidemiol. 2007;21:98-113. doi:10.1111/j.1365-3016.2007.00786.x

2. Rollins M, Lucero J. Overview of anesthetic considerations for Cesarean delivery. Br Med Bull. 2012;101:105-125. doi:10.1093/ $\mathrm{bmb} / \mathrm{ldr} 050$

3. Chinachoti T, Nilrat P, Samarnpiboonphol P. Nausea, vomiting and pruritus induced by intrathecal morphine. $J$ Med Assoc Thai. 2013;96:589-594.

4. Gehling M, Tryba M. Risks and side-effects of intrathecal morphine combined with spinal anaesthesia: a meta-analysis. Anaesthesia. 2009;64:643-651. doi:10.1111/j.1365-2044.2008.05817.x

5. Hindle A. Intrathecal opioids in the management of acute postoperative pain. BJA Educ. 2008;8:81-85.
6. Masood SN, Naim U, Masood MF. A randomized comparative trial of early initiation of oral maternal feeding versus conventional oral feeding after cesarean delivery. Int J Gynecol Obstet. 2014;126:115119. doi:10.1016/j.ijgo.2014.02.023

7. Bauer AJ, Boeckxstaens GE. Mechanisms of postoperative ileus. Neurogastroenterol Motil. 2004;16:54-60. doi:10.1111/nmo.2004.16. issue-s2

8. Miedema BW, Johnson JO. Methods for decreasing postoperative gut dysmotility. Lancet Oncol. 2003;4:365-372.

9. Chapman SJ, Thorpe G, Vallance AE, et al. Systematic review of definitions and outcome measures for return of bowel function after gastrointestinal surgery. BJS Open. 2018;3(1):1-10. doi:10.1002/ bjs5.102

10. Jalilian N, Ghadami MR. Randomized clinical trial comparing postoperative outcomes of early versus late oral feeding after cesarean section. $J$ Obstet Gynaecol Res. 2014;40:1649-1652. doi:10.1111/jog.12246

11. Mehta S, Gupta S, Goel N. Postoperative oral feeding after cesarean section: early versus late initiation: a prospective randomized trial. $J$ Gynecol Surg. 2010;26:247-250. doi:10.1089/gyn.2009.0092

12. Izbizky GH, Minig L, Sebastiani MA, Otano L. The effect of early versus delayed postcaesarean feeding on women's satisfaction: a randomised controlled trial. BJOG. 2008;115:332-338. doi:10.1111/ j.1471-0528.2007.01591.x

13. Bar G, Sheiner E, Lezerovizt A, Lazer T, Hallak M. Early maternal feeding following caesarean delivery: a prospective randomised study. Acta Obstet Gynecol Scand. 2008;87:68-71. doi:10.1080/ 00016340701778849

14. Teoh WHL, Shah MK, Mah CL. A randomised controlled trial on beneficial effects of early feeding post-Caesarean delivery under regional anaesthesia. Singapore Med J. 2007;48:152-157.

15. Chantarasorn V, Tannirandorn Y. A comparative study of early postoperative feeding versus conventional feeding for patients undergoing cesarean section; a randomized controlled trial. J Med Assoc Thai. 2006;89(Suppl 4):11-16.

16. Kathpalia SK. Early maternal feeding versus traditional delayed feeding after cesarean section: a pilot study. $J$ Obstet Gynecol India. 2017;67:178-182. doi:10.1007/s13224-016-0949-0

17. Saad AF, Saoud F, Diken ZM, et al. Early versus late feeding after cesarean delivery: a randomized controlled trial. Am J Perinatol. 2016;33:415-419. doi:10.1055/s-0035-1565918

18. Huang H, Wang H, He M. Early oral feeding compared with delayed oral feeding after cesarean section: a meta-analysis. J Maternal Fetal Neonatal Med. 2016;29:423-429. doi:10.3109/14767058.2014.1002765

19. Guo J, Long S, Li H, et al. Early versus delayed oral feeding for patients after cesarean. Int J Gynaecol Obstet. 2015;128:100-105. doi:10.1016/j.ijgo.2014.07.039

20. Milton GW. Normal bowel sounds. S Afr J Surg. 1958;2:490-493.

21. Jamieson S. Likert scales: how to (Ab)use them. Med Educ. 2004;38:217-8. doi:10.1111/j.1365-2929.2004.02012.x

22. Keller D, Stein SL. Facilitating return of bowel function after colorectal surgery: alvimopan and gum chewing. Clin Colon Rectal Surg. 2013;26:186-190. doi:10.1055/s-0033-1351137

23. Habib AS, Wahl K, Gu J. Comparison of postoperative pain outcomes after vertical or Pfannenstiel incision for major gynecologic surgery. Curr Med Res Opin. 2009;25:1529-1534. doi:10.1185/03007990902959168
The International Journal of Women's Health is an international, peerreviewed open-access journal publishing original research, reports, editorials, reviews and commentaries on all aspects of women's healthcare including gynecology, obstetrics, and breast cancer. The manuscript management system is completely online and includes a very quick and fair peer-review system, which is all easy to use. Visit http://www.dovepress.com/testimonials.php to read real quotes from published authors. 